Research Article

\title{
The Quantum Symmetry in Nonbalanced Hopf Spin Models Determined by a Normal Coideal Subalgebra
}

\author{
Xin Qiaoling $\mathbb{D}^{1}$ and Cao Tianqing $\mathbb{D}^{2}$ \\ ${ }^{1}$ School of Mathematical Sciences, Tianjin Normal University, Tianjin 300387, China \\ ${ }^{2}$ School of Mathematical Sciences, Tiangong University, Tianjin 300387, China \\ Correspondence should be addressed to Xin Qiaoling; xinqiaoling0923@163.com \\ Received 1 March 2021; Revised 9 April 2021; Accepted 20 April 2021; Published 5 May 2021 \\ Academic Editor: Naihuan Jing \\ Copyright (๑) 2021 Xin Qiaoling and Cao Tianqing. This is an open access article distributed under the Creative Commons Attribution \\ License, which permits unrestricted use, distribution, and reproduction in any medium, provided the original work is properly cited. \\ For a finite-dimensional cocommutative semisimple Hopf $C^{*}$-algebra $H$ and a normal coideal $*$-subalgebra $H_{1}$, we define the \\ nonbalanced quantum double $D\left(H_{1} ; H\right)$ as the crossed product of $H$ with $H_{1}^{o p}$, with respect to the left coadjoint representation of \\ the first algebra acting on the second one, and then construct the infinite crossed product $\mathscr{A}_{H_{1}}=\cdots \rtimes H \rtimes \widehat{H_{1}} \rtimes H \rtimes \widehat{H_{1}} \rtimes H \rtimes \cdots$ as the \\ observable algebra of nonbalanced Hopf spin models. Under a right comodule algebra action of $D\left(H_{1} ; H\right)$ on $\mathscr{A}_{H_{1}}$, the field \\ algebra can be obtained as the crossed product $C^{*}$-algebra. Moreover, we prove there exists a duality between the nonbalanced \\ quantum double $D\left(H_{1} ; H\right)$ and the observable algebra $\mathscr{A}_{H_{1}}$.
}

\section{Introduction}

A model consists of a mathematical description of the system and the energy function. Quantum chains as low-dimensional $(1+1)$ models possess many interesting features, such as integrability and quantum symmetry. One of the simplest examples exhibiting quantum symmetry is $G$-spin models, introduced by K. Szlachanyi and P. Vecsernyes [1] in lattice field theories, where $G$ is a finite group. $G$-spin models as a classical statistical systems have an order-disorder type of quantum symmetry, which is the quantum double $D(G)$. The quantum symmetry in $G$-spin models generalizes the $\mathbb{Z}_{2} \times \mathbb{Z}_{2}$ symmetry which can sharply divide the ordered phase and the disordered phase in Ising models. Generally, if $G$ is an Abelian group, $G$-spin models have a symmetry group $G \times \widehat{G}$, which is the direct product of the group $G$ and the group of characters of $G$. Based on a field-theory analysis of $G$-spin models, Jiang and Guo [2] gave the concrete construction of a $D(G ; N)$-invariant subspace in field algebra of $G$-spin models and proved the $D(G ; N)$-invariant subspace is Galois closed if $N$ is a normal subgroup of $G$. On the contrary, Xin and Jiang [3] generalized $G$-spin models to $G$-spin models determined by a normal subgroup $N$, in which the quantum double $D(N ; G)$, and the field algebra determined by $N$ are defined, and then, the observable algebra determined by $N$ can be obtained as $D(N ; G)$-invariant subalgebra. Based on these work, the quantum symmetry is given by the quantum double $D(N ; G)$.

Note that $\widehat{G}$, the disorder part of the quantum double $D(G)$, is Abelian, so $G$-spin models do not have Kramers-Wannier duality for non-Abelian group. For this reason, $G$-spin models can be extended to a larger class of models. In 1997, F. Nill and K. Szlachanyi [4] investigated a one-dimensional quantum chains of Hopf algebras, called Hopf spin models, i.e., for a finite dimensional Hopf $C^{*}$-algebra $H$, there is a copy of $H$ on every lattice and a copy of $\widehat{H}$ on every link, and they satisfy nontrivial commutation relations only if they are neighbour links and sites, where $\widehat{H}$ is the dual of $H$. The two-side infinite crossed product is defined as the observable algebra of Hopf spin models, and the field algebra is the crossed product of the observable algebra by the comodule action. Subsequently, the authors [5] considered the Jones basic construction on Hopf spin models and constructed the crossed product of the field algebra by the dual of the quantum double for Hopf algebra, which is consistent with Jones basic construction for the field algebra and the observable algebra. In this paper, we consider the more general situation. For a finitedimensional cocommutative semisimple Hopf $C^{*}$-algebra $H$ and a normal coideal $*$-subalgebra $H_{1}$, we define the quantum 
double $D\left(H_{1} ; H\right)$ by means of the left $H$-module algebra structure on Hopf $C^{*}$-algebra $\widehat{H_{1}^{o p}}$, where $\widehat{H_{1}^{o p}}$ is the dual of the opposite Hopf $C^{*}$-algebra of $H_{1}$. Since Hopf $C^{*}$-algebras on both sides of $\otimes$ in the quantum double $D\left(H_{1} ; H\right)$ are different, we call $D\left(H_{1} ; H\right)$ the nonbalanced quantum double. In particular, let $H=\mathbb{C}(G)$ be the group algebra of a finite group $G$ and $H_{1}=\mathbb{C} N$ be the group algebra of a normal subgroup $N$ of $G$; then, $H_{1}$ can be naturally regarded as the normal Hopf*subalgebra of $H$, and $D\left(H_{1} ; H\right)$ reduces to $D(N ; G)$ [3]. Moreover, we will also describe the quantum symmetry in the corresponding quantum chains of $\mathrm{Hopf} \mathrm{C}^{*}$-algebras $\mathrm{H}$ and $\mathrm{H}_{1}$ called nonbalanced Hopf spin models: there is a copy of $\widehat{H}$ on every lattice site and a copy of $H_{1}$ on every link, and they satisfy some commutation relations, which generalizes the results established in $[3,4]$.

The paper is organized as follows. In Section 2, we introduce the nonbalanced quantum double and define a right comodule algebra action of nonbalanced quantum double $D\left(H_{1} ; H\right)$ on the observable algebra $\mathscr{A}_{H_{1}}$ determined by a semisimple normal Hopf ${ }^{*}$-subalgebra $H_{1}$. It is known that there is one-to-one correspondence between right $D\left(H_{1} ; H\right)$-comodule algebra actions and left $D\left(\widehat{H_{1}} ; H\right)$-module algebra actions, since $D\left(H_{1} ; H\right)$ is finite dimensional, where $D\left(\widehat{H_{1}} ; H\right)$ is the dual of $D\left(H_{1} ; H\right)$. Based on these, we can define the field algebra of nonbalanced Hopf spin models as the crossed product $C^{*}$-algebra of the observable algebra $\mathscr{A}_{H_{1}}$ by $D\left(\widehat{H_{1}} ; H\right)$ in Definition 5 and prove that the observable algebra is the -invariant subspace of the field algebra. In Section 3, we prove that is the commutants of $\mathscr{A}_{(H, G)}$ and vice verse.

In this paper, we work over a complex field $\mathbb{C}$. All Hopf algebraic notations $[6,7]$ can be used in the following. For example, we denote by $m, \iota, S, \Delta, \mathcal{\varepsilon}$, * the multiplication, the unit, the antipode, the comultiplication, the counit, and *-operation, respectively. And, we use the standard notation $\Delta(a)=a_{(1)} \otimes a_{(2)} \quad$ and $\quad \Delta^{(n)}(a)=\Delta^{(n-1)} \circ(\operatorname{id} \otimes \Delta)(a)=$ $a_{(1)} \otimes a_{(2)} \otimes \cdots \otimes a_{(n+1)}$ in the Hopf algebra where there is an implicit summation on the right side and $\Delta \circ(\mathrm{id} \otimes \Delta)=\Delta \circ(\Delta \otimes \mathrm{id})$.

\section{The Field Algebra of Nonbalanced Hopf Spin Models}

2.1. The Nonbalanced Quantum Double. It is known that the quantum double $D(H)$, originally introduced by Drinfeld for a Hopf algebra $H$ [8], plays an important role in the field of mathematical physics, and the quasi-triangular structure leads to a braiding in the category of representations and many ensuing applications. In particular, when $H$ is the group algebra for a finite group, the quantum double reduces to an interesting crossed product algebra $C(G) \rtimes G$, where $C(G)$ denotes the algebra of complex valued functions on $G$ and the action is the conjugation [9]. Subsequently, several alternative descriptions of the quantum double have appeared in the literature. S. Majid [10] and F. Hausser and F. Nill $[11,12]$ independently introduced the quantum double of a finite dimensional quasi-Hopf algebra. In 2003,
D. Bulacu and S. Caenepeel [13] constructed the quantum double for quasi-triangular quasi-Hopf algebras. In 2004, L. Delvaux and A. Van Daele [14] gave the Drinfeld double of multiplier Hopf algebras, which generalized the usual quantum double for Hopf algebras. In the usual quantum double $D(H)=\widehat{H^{o P}} \otimes H$, two (generalized) Hopf $C^{*}$-algebra on both sides of $\otimes$ are the same. In this section, we hope to consider the similar theory when Hopf $C^{*}$-algebra are different. For this aim, we will generalize the quantum double to the nonbalanced quantum double by replacing $D(H)$ by $D\left(H_{1} ; H\right)=\overline{H_{1}^{o p}} \otimes H$, where $H_{1}$ is a normal Hopf *-subalgebra of $H$. In [15], Chen considered the nonbalanced quantum double $D\left(H_{1} ; H\right)=\widehat{H_{1}^{o p}} \otimes H$, where $H$ is a finite-dimensional Hopf algebra and $H_{1}$ is a Hopf subalgebra of $H$, and showed $D\left(H_{1} ; H\right)$ is a Hopf algebra. On the basis of the work, we will prove that $D\left(H_{1} ; H\right)$ is a Hopf $C^{*}$-algebra by using the way different from [15]. Let us recall the following definition.

Definition 1. Let $M$ be a *-algebra and $A$ be a Hopf $C^{*}$-algebra. Then, $M$ is a left $A$-module algebra if there is a left action $\triangleright: A \otimes M \longrightarrow M,(a \otimes m) \mapsto a \triangleright m$, and satisfies the following conditions, for any $a, b \in A, m, n \in M$ :

$$
\begin{aligned}
(a b) \triangleright m & =a \triangleright(b \triangleright m), \\
1_{A} \triangleright m & =m, \\
a \triangleright(m n) & =\left(a_{(1)} \triangleright m\right)\left(a_{(2)} \triangleright n\right), \\
a \triangleright m^{*} & =\left(S a^{*} \triangleright m\right)^{*}, \\
a \triangleright 1_{M} & =\varepsilon(a) 1_{M} .
\end{aligned}
$$

If $M$ is a $C^{*}$-algebra, the map $a \triangleright$ is assumed to be norm continuous for all $a \in A$.

Theorem 1. If $M$ is a left A-module algebra, the crossed product $M \rtimes A$ is a *-algebra with the *-algebra structure given for $m, n \in M, a, b \in A$ by

$$
\begin{aligned}
(m \otimes a)(n \otimes b) & =\left(m a_{(1)} n \otimes a_{(2)} b\right), \\
(m \otimes a)^{*} & =\left(1_{M} \otimes a^{*}\right)\left(m^{*} \otimes 1_{A}\right) .
\end{aligned}
$$

Moreover, if $M$ is a $C^{*}$-algebra and $A$ is of finite dimension, then the crossed product $M \rtimes A$ becomes a $C^{*}$-algebra.

Proof. Clearly, $M \rtimes A$ has the *-algebra structure. We will show that $M \rtimes A$ is a $C^{*}$-algebra in the following.

It follows from [16] that $M$ has a faithful positive linear functional $\varphi_{M}$ and $A$ has an invariant functional $\varphi_{A}$ such that

$$
\varphi_{A}=\varphi_{A} \circ S \text { and }\left(\varphi_{A} \otimes \mathrm{id}\right) \Delta(a)=\left(\mathrm{id} \otimes \varphi_{A}\right) \Delta(a)=\varphi_{A} 1_{A},
$$

for all $a \in A$. Define the map $\theta$ on $M \rtimes A$ as follows:

$$
\theta(m \otimes a)=\varphi_{M}(m) \varphi_{A}(a) .
$$

Then, $\theta$ is a faithful positive linear functional on $M \rtimes A$. In fact, 


$$
\begin{aligned}
\theta\left((m \otimes a)(m \otimes a)^{*}\right) & =\theta\left((m \otimes a)\left(a_{(1)}^{*} m^{*} \otimes a_{(2)}^{*}\right)\right) \\
& =\theta\left(m a_{(1)} a_{(1)}^{*} m^{*} \otimes a_{(2)} a_{(2)}^{*}\right)=\theta\left(m a_{(1)} a_{(1)}^{*} m^{*} \otimes a_{(2)} a_{(2)}^{*}\right) \\
& =\varphi_{M}\left(m a_{(1)} a_{(1)}^{*} m^{*}\right) \varphi_{A}\left(a_{(2)} a_{(2)}^{*}\right)=\varphi_{M}\left(m a_{(1)} a_{(1)}^{*} \varphi_{A}\left(a_{(2)} a_{(2)}^{*}\right) m^{*}\right) \\
& =\varphi_{M}\left(m \varphi_{A}\left(a a^{*}\right) 1_{A} m^{*}\right)=\varphi_{M}\left(m m^{*}\right) \varphi_{A}\left(a a^{*}\right) \geq 0,
\end{aligned}
$$

and $\theta\left((m \otimes a)(m \otimes a)^{*}\right)=0$ if and only if $m \otimes a=0$.

By [17], we can construct the associated GNS representation of $M \rtimes A$. Denote by $\mathscr{K}$ the completion of $M \rtimes A$ with respect to the inner product $\langle m, x\rangle=\theta\left(x^{*} m\right)$. Let $\tau: M \rtimes A \longrightarrow B(\mathscr{K})$ be the left multiplication; then, $M \rtimes A$ can embed isometrically into $B(K)$, and thus, it is a $C^{*}$-algebra.

Semisimple Hopf algebras are intensively studied since they have important applications in topological invariants of knots and manifolds, quantum field theory, and so on. In fact, a Hopf algebra can be recovered from a normal Hopf subalgebra and some additional cohomological data [18]. As a result, normal Hopf subalgebras are an important tool in the classification of semisimple Hopf algebras. In 2012, B. Sebastian [19] studied normal left coideal subalgebras of semisimple Hopf algebras. In 2020, the authors [20] proved that, for a semisimple Hopf algebra, there is a one-to-one correspondence between right group-like projections and left coideal subalgebras.

Definition 2. Let $H$ be any finite-dimensional semisimple Hopf $C^{*}$-algebra:

(1) A coideal subalgebra $K$ of $H$ is a subalgebra $K$ of $H$ with $\Delta(K) \subseteq H \otimes K+K \otimes H$.

(2) A Hopf subalgebra $K$ of $H$ is said to be normal if $K$ is invariant with respect to the right and left adjoint action:

$$
\begin{aligned}
& \left(S h_{(1)}\right) k h_{(2)} \in K, \\
& h_{(1)} k\left(S h_{(2)}\right) \in K,
\end{aligned}
$$

for all $h \in H, k \in K$.
(3) A coideal subalgebra $K$ which is also a normal *-subalgebra of $H$ is said to be normal coideal *-subalgebra of $H$.

Remark 1

(1) If $K$ is a coideal subalgebra, then $K$ is also a semisimple Hopf subalgebra (see Lemma 4.0.2 in [21]).

(2) Suppose that $G$ is a finite group and $N$ is a normal subgroup of $G$. In this case of $H=\mathbb{C} G$, the group algebra of $G, K=\mathbb{C N}$ can be viewed as a normal coideal $*$-subalgebra of $H$.

Note that the quantum double $D(G)$ of a finite group is the crossed product of $C(G)$ by the group algebra $\mathbb{C} G$ in $G$-spin models, and $\mathbb{C} G$ is always cocommutative. In order to give the definition of the nonbalanced quantum double in nonbalanced Hopf spin models, we suppose that $H$ is a cocommutative semisimple Hopf $C^{*}$-algebra of finite dimension and $H_{1}$ is a normal coideal $*$-subalgebra of $H$ in the following. The left coadjoint representation of $H$ on the dual of opposite Hopf $C^{*}$-algebra $\widehat{H_{1}^{o p}}$ is given for $a \in H_{1}, x \in H$, and $f \in \widehat{H_{1}}$ by

$$
\langle a, x \cdot f\rangle=\left\langle S\left(x_{(2)}\right) a x_{(1)}, f\right\rangle,
$$

where $\langle\cdot, \cdot\rangle$ denotes the canonical pairing between $H_{1}$ and $\widehat{H_{1}}$.

Proposition 1. The Hopf $C^{*}$-algebra $\widehat{H_{1}^{o p}}$ is a left H-module algebra for the left coadjoint representation.

Proof. Suppose that $x, y \in H, f, g \in \widehat{H_{1}^{o p}}$, and $a \in H_{1}$; then, we have

$$
\langle a,(x y) \cdot f\rangle=\left\langle S(x y)_{(2)} a(x y)_{(1)}, f\right\rangle=\left\langle S y_{(2)} S x_{(1)} a x_{(1)} y_{(1)}, f\right\rangle=\left\langle S x_{(1)} a x_{(1)}, y \cdot f\right\rangle=\langle a, x \cdot(y \cdot f)\rangle .
$$

Hence, $(x y) \cdot f=x \cdot(y \cdot f)$, which means that $\widehat{H_{1}^{o p}}$ is a left $H$-module. Subsequently, using the cocommutativity of $H$, we obtain

$$
\begin{aligned}
\langle a, x \cdot(f g)\rangle & =\left\langle S x_{(2)} a x_{(1)}, f g\right\rangle=\left\langle\left(S x_{(2)} a x_{(1)}\right)_{(1)}, f\right\rangle\left\langle\left(S x_{(2)} a x_{(1)}\right)_{(2)}, g\right\rangle=\left\langle S x_{(4)} a_{(1)} x_{(1)}, f\right\rangle\left\langle S x_{(3)} a_{(2)} x_{(2)}, g\right\rangle \\
& =\left\langle S x_{(2)} a_{(1)} x_{(2)}, f\right\rangle\left\langle S x_{(4)} a_{(2)} x_{(3)}, g\right\rangle=\left\langle a_{(1)}, x_{(1)} \cdot f\right\rangle\left\langle a_{(2)}, x_{(2)} \cdot g\right\rangle=\left\langle a,\left(x_{(1)} \cdot f\right)\left(x_{(2)} \cdot g\right)\right\rangle .
\end{aligned}
$$


In order to complete the proof, we have to check $x \cdot f^{*}=\left(S x^{*} \cdot f\right)^{*}$. Since $*$-structure of $\widehat{H}$ is defined by $\left\langle a, f^{*}\right\rangle=\overline{\left\langle(S a)^{*}, f\right\rangle}$, then

$$
\begin{aligned}
\left\langle a, x \cdot f^{*}\right\rangle & =\left\langle S x_{(2)} a x_{(1)}, f^{*}\right\rangle=\overline{\left\langle\left(S\left(S x_{(2)} a x_{(1)}\right)\right)^{*}, f\right\rangle}=\overline{\left\langle\left(S x_{(1)} S a x_{(2)}\right)^{*}, f\right\rangle} \\
& =\overline{\left\langle x_{(2)}^{*}(S a)^{*}\left(S x_{(1)}\right)^{*}, f\right\rangle}=\overline{\left\langle x_{(2)}^{*} S a^{*} S x_{(1)}^{*}, f\right\rangle}=\overline{\left\langle S\left(S x^{*}\right)_{(2)}(S a)^{*}\left(S x^{*}\right)_{(1)}, f\right\rangle} \\
& =\overline{\left\langle(S a)^{*}, S x^{*} \cdot f\right\rangle}=\left\langle a,\left(S x^{*} \cdot f\right)^{*}\right\rangle,
\end{aligned}
$$

where we use the fact $S^{2}=$ id and $S$ is an anti-coalgebramorphism [7].

Definition 3. The nonbalanced quantum double $D\left(H_{1} ; H\right)$ of $H$ and $H_{1}$ is defined as the crossed product of $H$ with $\overline{H_{1}^{o p}}$, with respect to the left coadjoint representation of the first algebra acting on the second one:

$$
D\left(H_{1} ; H\right)=\widehat{H_{1}^{o p}} \rtimes H .
$$

The Hopf *-structures are as follows, for $f \otimes x, g \otimes y \in D D\left(H_{1} ; H\right):$

$$
\begin{aligned}
& (f \otimes x)(g \otimes y)=f\left(x_{(2)}-g \leftarrow S\left(x_{(1)}\right)\right) \otimes x_{(3)} y, \text { (multiplication), } \\
& 1_{D\left(H_{1} ; H\right)}=\varepsilon_{H_{1}} \otimes 1_{H} \text {, (unit), } \\
& \Delta(f \otimes x)=\left(f_{(2)} \otimes x_{(1)}\right) \otimes\left(f_{(1)} \otimes x_{(2)}\right), \text { (comultiplication), } \\
& \varepsilon(f \otimes x)=f\left(1_{H_{1}}\right) \varepsilon(x) \text {, (counit), } \\
& S(f \otimes x)=\left(S\left(x_{(2)}\right) \longrightarrow S(f) \leftarrow x_{(1)}\right) \otimes S\left(x_{(3)}\right), \text { (antipode), } \\
& (f \otimes x)^{*}=\left(x_{(2)}^{*} \rightarrow f^{*} \leftarrow S\left(x_{(1)}^{*}\right)\right) \otimes x_{(3)}^{*},(*-\text { operation }),
\end{aligned}
$$

where Sweedler's arrows $\rightarrow(\longleftarrow)$ denote the transpose of right (left) multiplication.

Remark 2. $D\left(H_{1} ; H\right)$ is semisimple. Indeed, since $H_{1}$ is semisimple, so is $\widehat{H_{1}^{o p}}$ [22]. Let $T$ and $t$ be a unique integral in
$\widehat{H_{1}^{o p}}$ and $H$ such that $\mathcal{\varepsilon}_{H_{1}^{o p}}(T)=1$ and $\varepsilon_{H}(t)=1$; then, $\varepsilon_{D\left(H_{1} ; H\right)}(T \otimes t)=\varepsilon \widehat{\mathcal{H}}_{1}^{\text {op }}(T) \varepsilon_{H}(t)=1$, and

$$
\begin{aligned}
(T \otimes t)(f \otimes x) & =T\left(t_{(2)}-f<S\left(t_{(1)}\right)\right) \otimes t_{(3)} x=T\left\langle f_{(1)}, S\left(t_{(1)}\right)\right\rangle\left\langle f_{(3)}, t_{(2)}\right\rangle \varepsilon\left(f_{(2)}\right) \otimes t_{(3)} x \\
& =T\left\langle f_{(1)}, S\left(t_{(1)}\right)\right\rangle\left\langle f_{(2)}, t_{(2)}\right\rangle \otimes t_{(3)} x=T \varepsilon\left(t_{(1)}\right) f\left(1_{H_{1}}\right) \otimes t_{(2)} x=T f\left(1_{H_{1}}\right) \otimes t x \\
& =T f\left(1_{H_{1}}\right) \otimes t \varepsilon(x)=\varepsilon(f \otimes x)(T \otimes t) .
\end{aligned}
$$

Maschke theorem tells that any finite dimensional Hopf algebra $K$ is semisimple iff there is a nonzero integral $\lambda \in K$ such that $\varepsilon_{K}(\lambda) \neq 0$. Hence, $D\left(H_{1} ; H\right)$ is semisimple.

2.2. The Observable Algebra and the Field Algebra. Let us continue to assume that $H$ is a finite-dimensional cocommutative semisimple Hopf $C^{*}$-algebra and $H_{1}$ is a normal coideal $*$-subalgebra of $H$. Consider 1 -dimensional lattice, which is composed of the lattice sites and links. We use even (odd) integers to denote lattice sites (links). There is a copy $A_{2 i}$ of $H$ on each lattice site and a copy $A_{2 i+1}$ of $\widehat{H_{1}}$, the dual of $H_{1}$ on each link.
Definition 4 . The quasi-local observable algebra $\mathscr{A}_{H_{1}}^{\text {loc }}$ of nonbalanced Hopf spin models determined by the normal Hopf *-subalgebra $H_{1}$ is a unital algebra generated by $\left\{A_{2 i}(a), A_{2 i+1}(\varphi): a \in H, \varphi \in \widehat{H_{1}}, i \in \mathbb{Z}\right\}$ subject to

$$
\begin{aligned}
A B & =B A, \quad A \in A_{i}, B \in A_{j},|i-j| \geq 2, \\
A_{2 i+1}(\varphi) A_{2 i}(a) & =A_{2 i}\left(a_{(1)}\right)\left\langle a_{(2)}, \varphi_{(1)}\right\rangle A_{2 i+1}\left(\varphi_{(2)}\right), \\
A_{2 i}(a) A_{2 i-1}(\varphi) & =A_{2 i-1}\left(\varphi_{(1)}\right)\left\langle\varphi_{(2)}, a_{(1)}\right\rangle A_{2 i}\left(a_{(2)}\right),
\end{aligned}
$$

where $\langle\cdot, \cdot\rangle$ denotes the canonical pairing between $H$ and $\hat{H}$.

Let $\mathscr{A}_{H_{1}}^{n, m}$ be a unital ${ }^{*}$-subalgebra of $\mathscr{A}_{H_{1}}^{\text {loc }}$ generated by $A_{i}, n<i<m$. Using the $C^{*}$-inductive limit [17], $\mathscr{A}_{H_{1}}^{\text {loc }}$ can be 
extended to a $C^{*}$-algebra $\mathscr{A}_{H_{1}}$ called the observable algebra of nonbalanced Hopf spin models determined by a normal coideal $*$-subalgebra $H_{1}$.
Proposition 2. For an interval $\Lambda$ of length 2, the map $\rho_{\Lambda}: \mathscr{A}_{H_{1}}^{\Lambda} \longrightarrow \mathscr{A}_{H_{1}}^{\Lambda} \otimes D\left(H_{1} ; H\right)$ given by

$$
\begin{aligned}
& \rho_{2 i-1,2 i}\left(A_{2 i-1}(\varphi) A_{2 i}(a)\right)=A_{2 i-1}\left(\varphi_{(1)}\right) A_{2 i}\left(a_{(2)}\right) \otimes\left(\varphi_{(2)} \otimes a_{(1)}\right), \\
& \rho_{2 i, 2 i+1}\left(A_{2 i}(a) A_{2 i+1}(\varphi)\right)=A_{2 i}\left(a_{(1)}\right) A_{2 i+1}\left(\varphi_{(4)}\right) \otimes\left(\varphi_{(2)} \otimes a_{(3)}\right)\left\langle a_{(2)}, \varphi_{(3)}\right\rangle\left\langle S\left(a_{(4)}\right), \varphi_{(1)}\right\rangle,
\end{aligned}
$$

where $a \in H$ and $\varphi \in \widehat{H_{1}}$ define a right comodule algebra action of $\mathrm{D}\left(H_{1} ; H\right)$ on $\mathscr{A}_{H_{1}}^{2 i-1,2 i}\left(\mathscr{A}_{H_{1}}^{2 i, 2 i+1}\right)$ with respect to $\Delta^{o p}$ $(\Delta)$.

Proof. Let us show that $\mathscr{A}_{H_{1}}^{2 i-1,2 i}$ is a right $D\left(H_{1} ; H\right)$-comodule algebra with respect to $\rho_{2 i-1,2 i}$. It suffices to check that the map $\rho_{2 i-1,2 i}$ satisfies the following relations:

$$
\begin{aligned}
\left(\rho_{2 i-1,2 i} \otimes \mathrm{id}\right) \circ \rho_{2 i-1,2 i} & =\left(\mathrm{id} \otimes \Delta^{o p}\right) \circ \rho_{2 i-1,2 i}, \\
(\mathrm{id} \otimes \varepsilon) \circ \rho_{2 i-1,2 i} & =\mathrm{id}, \\
\rho_{2 i-1,2 i} \circ m & =m \circ\left(\rho_{2 i-1,2 i} \otimes \rho_{2 i-1,2 i}\right), \\
\rho_{2 i-1,2 i}\left(1_{\mathscr{A}_{H_{1}}^{2 i-1,2 i}}\right) & =1_{\mathscr{A}_{H_{1}}^{2 i-1,2 i}} \otimes 1_{D\left(H_{1} ; H\right)} .
\end{aligned}
$$

Now, let us compute by evaluating both sides on an element $A_{2 i-1}(\varphi) A_{2 i}(a)$ in $\mathscr{A}_{H_{1}}^{2 i-1,2 i}$ :

$$
\begin{aligned}
\left(\rho_{2 i-1,2 i} \otimes \mathrm{id}\right) \circ \rho_{2 i-1,2 i}\left(A_{2 i-1}(\varphi) A_{2 i}(a)\right) & =\rho_{2 i-1,2 i}\left(A_{2 i-1}\left(\varphi_{(1)}\right) A_{2 i}\left(a_{(2)}\right)\right) \otimes\left(\varphi_{(2)} \otimes a_{(1)}\right) \\
& =A_{2 i-1}\left(\varphi_{(1)}\right) A_{2 i}\left(a_{(3)}\right) \otimes\left(\varphi_{(2)} \otimes a_{(2)}\right) \otimes\left(\varphi_{(3)} \otimes a_{(1)}\right)=A_{2 i-1}\left(\varphi_{(1)}\right) A_{2 i}\left(a_{(2)}\right) \otimes \Delta\left(\varphi_{(2)} \otimes a_{(1)}\right) \\
& =\left(\operatorname{id} \otimes \Delta^{o p}\right) \circ \rho_{2 i-1,2 i}\left(A_{2 i-1}(\varphi) A_{2 i}(a)\right), \\
(\mathrm{id} \otimes \varepsilon) \circ \rho_{2 i-1,2 i}\left(A_{2 i-1}(\varphi) A_{2 i}(a)\right) & =A_{2 i-1}\left(\varphi_{(1)}\right) A_{2 i}\left(a_{(2)}\right) \otimes \varepsilon\left(\varphi_{(2)} \otimes a_{(1)}\right) \\
& =A_{2 i-1}\left(\varphi_{(1)}\right) A_{2 i}\left(a_{(2)}\right) \otimes \varphi_{(2)}\left(1_{H}\right) \varepsilon\left(a_{(1)}\right)=A_{2 i-1}(\varphi) A_{2 i}(a) .
\end{aligned}
$$

Hence, $\mathscr{A}_{H_{1}}^{2 i-1,2 i}$ is a right $D\left(H_{1} ; H\right)$-comodule. It remains to prove that $\rho_{2 i-1,2 i}$ is a $*$-algebra homomorphism. Using the commutative relations in Definition 4 , we obtain

$$
\begin{aligned}
& \rho_{2 i-1,2 i}\left(A_{2 i-1}(\varphi) A_{2 i}(a) A_{2 i-1}(\psi) A_{2 i}(b)\right) \\
& =\rho_{2 i-1,2 i}\left\langle A_{2 i-1}(\varphi) A_{2 i-1}\left(\psi_{(1)}\right)\left\langle\psi_{(2)}, a_{(1)}\right\rangle A_{2 i}\left(a_{(2)}\right) A_{2 i}(b)\right\rangle \\
& =\rho_{2 i-1,2 i}\left(A_{2 i-1}\left(\varphi \psi_{(1)}\right)\left\langle\psi_{(2)}, a_{(1)}\right\rangle A_{2 i}\left(a_{(2)} b\right)\right) \\
& =A_{2 i-1}\left(\varphi \psi_{(1)}\right)_{(1)} A_{2 i}\left(a_{(2)} b\right)_{(2)}\left\langle\psi_{(2)}, a_{(1)}\right\rangle \otimes\left(\varphi \psi_{(1)}\right)_{(2)} \otimes\left(a_{(2)} b\right)_{(1)} \\
& =A_{2 i-1}\left(\varphi_{(1)} \psi_{(1)}\right) A_{2 i}\left(a_{(3)} b_{(2)}\right)\left\langle\psi_{(3)}, a_{(1)}\right\rangle \otimes \varphi_{(2)} \psi_{(2)} \otimes a_{(2)} b_{(1)} .
\end{aligned}
$$

Similarly, we have

$$
\begin{aligned}
& \rho_{2 i-1,2 i}\left(A_{2 i-1}(\varphi) A_{2 i}(a)\right) \rho_{2 i-1,2 i}\left(A_{2 i-1}(\psi) A_{2 i}(b)\right) \\
& =A_{2 i-1}\left(\varphi_{(1)}\right) A_{2 i}\left(a_{(2)}\right) A_{2 i-1}\left(\psi_{(1)}\right) A_{2 i}\left(b_{(2)}\right) \otimes\left(\varphi_{(2)} \otimes a_{(1)}\right)\left(\psi_{(2)} \otimes b_{(1)}\right) \\
& =A_{2 i-1}\left(\varphi_{(1)}\right) A_{2 i-1}\left(\psi_{(1)}\right)\left\langle\psi_{(2)}, a_{(4)}\right\rangle A_{2 i}\left(a_{(5)}\right) A_{2 i}\left(b_{(2)}\right) \otimes \varphi_{(2)} \psi_{(4)}\left\langle\psi_{(5)}, a_{(2)}\right\rangle\left\langle\psi_{(3)}, S a_{(1)}\right\rangle \otimes a_{(3)} b_{(1)} \\
& =A_{2 i-1}\left(\varphi_{(1)} \psi_{(1)}\right) A_{2 i}\left(a_{(5)} b_{(2)}\right) \otimes \varphi_{(2)} \psi_{(4)} \otimes a_{(4)} b_{(1)}\left\langle\psi_{(5)}, a_{(1)}\right\rangle\left\langle\psi_{(2)}, a_{(2)}\right\rangle\left\langle\psi_{(3)}, S a_{(3)}\right\rangle \\
& =A_{2 i-1}\left(\varphi_{(1)} \psi_{(1)}\right) A_{2 i}\left(a_{(4)} b_{(2)}\right) \otimes \varphi_{(2)} \psi_{(3)} \otimes a_{(3)} b_{(1)}\left\langle\psi_{(4)}, a_{(1)}\right\rangle\left\langle\psi_{(2)}, \varepsilon\left(a_{(2)}\right) 1_{H}\right\rangle \\
& =A_{2 i-1}\left(\varphi_{(1)} \psi_{(1)}\right) A_{2 i}\left(a_{(3)} b_{(2)}\right) \otimes \varphi_{(2)} \psi_{(2)} \otimes a_{(2)} b_{(1)}\left\langle\psi_{(3)}, a_{(1)}\right\rangle,
\end{aligned}
$$


where we use the cocommutativity of $H$ and $\sum_{(a)} a_{(1)}\left(a_{(2)} S a_{(3)}\right)=\sum_{(a)} a_{(1)} \varepsilon\left(a_{(2)}\right) 1_{H}=a$.

By induction, we can define a comodule algebra action of $D\left(H_{1} ; H\right)$ on $\mathscr{A}_{H}^{\Lambda}$ for any finite interval $\Lambda \subset \mathbb{Z}$. It is known that if $\mathscr{A}_{H_{1}}^{\Lambda}$ is a right $D\left(H_{1} ; H\right)$-comodule algebra, then $\mathscr{A}_{H_{1}}^{\Lambda}$ is a left $D\left(\widehat{H_{1}} ; H\right)$-module algebra via

$$
\begin{aligned}
\rho_{\xi}: \mathscr{A}_{H_{1}}^{\Lambda} & \longrightarrow \mathscr{A}_{H_{1}}^{\Lambda}, \\
A & \mapsto\left(\operatorname{id}_{\mathscr{A}} \otimes \xi\right)(\rho(A)),
\end{aligned}
$$

for any $\xi \in D\left(\widehat{H_{1}} ; H\right), A \in \mathscr{A}_{H_{1}}^{\Lambda}$, where $D\left(\widehat{H_{1}} ; H\right)$ is the dual of $D\left(H_{1} ; H\right)$. By Theorem 1 , we can obtain the crossed product $C^{*}$-algebra of finite dimension $\mathscr{A}_{H_{1}}^{\Lambda} \rtimes D\left(\widehat{H_{1}} ; H\right)$, denoted by $\mathscr{F}_{H_{1}}^{\Lambda}$. Let $\Lambda_{n}$ be an increasing sequence of intervals; then, the natural embeddings $\iota_{n}: \mathscr{F}_{H_{1}} \Lambda_{n} \longrightarrow \mathscr{F}_{H_{1}}^{\Lambda_{n+1}}$ are norm preserving.

Definition 5. On the nonbalanced Hopf spin models, the field algebra $\mathscr{F}_{H_{1}}$ is defined as the $C^{*}$-inductive limit for finite dimensional $C^{*}$-algebras $\mathscr{F}_{H_{1}}^{\Lambda_{n}}$.

Using the uniqueness of $C^{*}$-inductive limit, the field algebra $\mathscr{F}_{H_{1}}$ is actually the crossed product $C^{*}$-algebra $\mathscr{A}_{H_{1}} \rtimes D\left(\widehat{H_{1}} ; H\right)$ with respect to the comodule algebra. For convenience, denote by $(A, \xi)$ the generating element in $\mathscr{F}_{H_{1}}$.

For any $X \in D\left(H_{1} ; H\right)$ and $(A, \xi) \in \mathscr{F}_{H_{1}}$, we define $\alpha: D\left(H_{1} ; H\right) \times \mathscr{F}_{H_{1}} \longrightarrow \mathscr{F}_{H_{1}}$ given by

$$
\alpha(X \otimes(A, \xi))=\left(A, \xi_{(1)}\right)\left\langle X, \xi_{(2)}\right\rangle .
$$

From now on, we suppress $\alpha$ and write $X(A, \xi)$ for $\alpha(X \otimes(A, \xi))$.

Proposition 3. The field algebra $\mathscr{F}_{H_{1}}$ determined by a normal Hopf *-subalgebra $H_{1}$ is a left $D\left(H_{1} ; H\right)$-module algebra.

Proof. This follows from straightforward computations.
Remark 3

(1) In Remark 2, we have shown that $T \otimes t$ is the unique integral in $D\left(H_{1} ; H\right)$. Moreover, we have the following fact:

$$
\mathscr{A}_{H_{1}}=\left\{F \in \mathscr{F}_{H_{1}}:(T \otimes t) F=F\right\} .
$$

(2) Let $H_{1}$ and $H_{2}$ be normal cosemisimple Hopf *-subalgebras of $H$. If $H_{1} \subseteq H_{2}$, then $\mathscr{A}_{H_{1}} \subseteq \mathscr{A}_{H_{2}}$.

\section{Quantum Double Symmetry}

The main objective of this section is to build a duality between the quantum double $D\left(H_{1} ; H\right)$ and the observable algebra defined in Section 2.

Theorem 2. Suppose that $\Pi$ is an irreducible representation of $\mathscr{F}_{H_{1}}$ on the Hilbert space $\mathscr{H}=\overline{\Pi\left(\mathscr{F}_{H_{1}}\right) \Omega}$ with a vacuum vector $\Omega$ satisfying

$$
(\Omega, \Pi(X(F)) \Omega)=\varepsilon(X)(\Omega, \pi(F) \Omega), \quad \forall X \in D\left(H_{1} ; H\right), F \in \mathscr{F}_{H_{1}} .
$$

Then, there is a unique $C^{*}$-homomorphism $U$ from $D\left(H_{1} ; H\right)$ to $B(\mathscr{H})$ such that

(1) $U\left(X_{(1)}\right) \Pi(F) U\left(S X_{(2)}\right)=$

$\Pi(X(F)), \forall X \in D\left(H_{1} ; H\right), \forall F \in \mathscr{F}_{H_{1}}$

(2) $\left(U\left(D\left(H_{1} ; H\right)\right)\right)^{\prime}=\overline{\Pi\left(\mathscr{A}_{H_{1}}\right)}$,

$U\left(D\left(H_{1} ; H\right)\right)=\Pi\left(\mathscr{A}_{H_{1}}\right)^{\prime}$, where the prime means commutant in $B(\mathscr{H})$ and the bar denotes weak closure

Proof

(1) For any $X \in D\left(H_{1} ; H\right)$, the map $U(X): \Pi\left(\mathscr{F}_{H_{1}}\right) \Omega \longrightarrow \Pi\left(\mathscr{F}_{H_{1}}\right) \Omega$ is defined by

$U(X)(\Pi(F) \Omega)=\Pi(X(F)) \Omega, \quad \forall F \in \mathscr{F}_{H_{1}}$.

Firstly, it is well-defined. In fact, for any $T, F \in \mathscr{F}_{H_{1}}$ and $X \in D\left(H_{1} ; H\right)$, we have

$$
\begin{aligned}
(\Pi(T) \Omega, \Pi(X(F)) \Omega) & =\left(\Omega, \Pi\left(T^{*}(X(F))\right) \Omega\right)=\left(\Omega, \Pi\left(T^{*}\left(X_{(1)}(F)\right)\right) \Omega\right) \varepsilon\left(S X_{(2)}\right) \\
& =\left(\Omega, \Pi\left(S X_{(2)}\left(T^{*}\left(X_{(1)}(F)\right)\right)\right) \Omega\right)=\left(\Omega, \Pi\left(S X_{(3)}\left(T^{*}\right)\left(S X_{(2)} X_{(1)}\right)(F)\right) \Omega\right) \\
& =\left(\Omega, \Pi\left(S X_{(2)}\left(T^{*}\right) \varepsilon\left(X_{(1)}\right)(F)\right) \Omega\right)=\left(\Pi\left(X^{*}(T)\right) \Omega, \Pi(F) \Omega\right) .
\end{aligned}
$$

which shows that $\Pi(F) \Omega=0$ can imply $\Pi(X(F)) \Omega=0$ for all $X \in D\left(H_{1} ; H\right)$.

Moreover, $U$ is a $*$-representation, i.e., $(U(X))^{*}=$ $U\left(X^{*}\right)$ and $U(X Y)=U(X) U(Y)$, which yields that
$\|U\| \leq 1$ from [23]. Hence, $U$ can be extended to $\mathscr{H}$ by continuity.

The uniqueness of $U$ is due to the fact $U(X) \Omega=\varepsilon(X) \Omega$. 
In order to check that $U\left(X_{(1)}\right) \Pi(F) U$ $\left(S X_{(2)}\right)=\Pi(X(F))$, it is enough to verify it on the generators $\Pi\left(\mathscr{F}_{H_{1}}\right) \Omega$ of the Hilbert space $\mathscr{H}$. For any $T \in \mathscr{F}_{H_{1}}$,

$$
\begin{aligned}
U\left(X_{(1)}\right) \Pi(F) U\left(S X_{(2)}\right)(\Pi(T) \Omega) & =U\left(X_{(1)}\right) \Pi\left(F\left(S X_{(2)}\right)\right)(T) \Omega \\
& =\Pi\left(X_{(1)}\left(F S X_{(2)}(T)\right)\right) \Omega=\Pi\left(X_{(1)}(F)\left(X_{(2)} S X_{(3)}\right)(T)\right) \Omega \\
& =\Pi\left(X_{(1)}(F)\right) \Pi\left(\varepsilon\left(X_{(2)}\right)(T)\right) \Omega=\Pi\left(X_{(1)} \varepsilon\left(X_{(2)}\right)(F)\right)(\Pi(T) \Omega)=\Pi(X(F))(\Pi(T) \Omega) .
\end{aligned}
$$

(2) It is enough to check that $\left(U\left(D\left(H_{1} ; H\right)\right)\right)^{\prime}$ $=\overline{\Pi\left(\mathscr{A}_{H_{1}}\right)}$, since any $C^{*}$-algebra of finite dimension is always weakly closed.

$$
\begin{aligned}
U(X) \Pi(F) \Pi(T) \Omega & =U(X) \Pi(F T) \Omega=\Pi\left(X_{(1)}(F) X_{(2)}(T)\right) \Omega \\
& =\Pi\left(\varepsilon\left(X_{(1)}\right)(F) X_{(2)}(T)\right) \Omega=\Pi(F X(T)) \Omega=\Pi(F) U(X) \Pi(T) \Omega,
\end{aligned}
$$

which shows $U(X) \Pi(F)=\Pi(F) U(X)$ for a dense subset $\Pi\left(\mathscr{F}_{H_{1}}\right) \Omega$ in $\mathscr{H}$. It follows from the continuity of $U$ that $U(X) \Pi(F)=\Pi(F) U(X) \quad$ in $\quad \mathscr{H}$. Thus, $\overline{\Pi\left(\mathscr{A}_{H_{1}}\right)} \subseteq(U$ $\left.\left(D\left(H_{1} ; H\right)\right)\right)^{\prime}$.

It remains to show that $\left(U\left(D\left(H_{1} ; H\right)\right)\right)^{\prime} \subseteq \overline{\Pi\left(\mathscr{A}_{H_{1}}\right)}$. Set $Z \in\left(U\left(D\left(H_{1} ; H\right)\right)\right)^{\prime}$; then,

$$
Z U(X)=U(X) Z, \quad \forall X \in D\left(H_{1} ; H\right) .
$$

Using the result [17], one can concludes that $\Pi\left(\mathscr{F}_{H_{1}}\right)$ is dense in $B(\mathscr{H})$ with respect to the weak operator topology, which yields that there exists a net $\left\{F_{\alpha}\right\} \subseteq \mathscr{F}_{H_{1}}$ such that $\Pi\left(F_{\alpha}\right)$ converges to $Z$, and then,

$$
\begin{array}{r}
U\left(X_{(1)}\right)\left(\Pi\left(F_{\alpha}\right)-Z\right) U\left(S X_{(2)}\right) \longrightarrow 0, \\
\Pi\left(X\left(F_{\alpha}\right)\right)-\varepsilon(X) Z \longrightarrow 0 .
\end{array}
$$

Let $X=T \otimes t$ be defined in Remark 2; then,

$$
\Pi\left(T \otimes t\left(F_{\alpha}\right)\right) \longrightarrow \varepsilon(T \otimes t) A=A,
$$

which means $\left(U\left(D\left(H_{1} ; H\right)\right)\right)^{\prime} \subseteq \overline{\Pi\left(\mathscr{A}_{(H, G)}\right)}$, and thus, $\left(U\left(D\left(H_{1} ; H\right)\right)\right)^{\prime}=\overline{\Pi\left(\mathscr{A}_{H_{1}}\right)}$.

\section{Data Availability}

No data were used to support this study.

\section{Conflicts of Interest}

The authors declare that they have no conflicts of interest.

\section{Acknowledgments}

The authors are greatly indebted to Jiang Lining for his helpful discussions. This work was supported by the National Natural Science Foundation of China (Grants nos. 11701423, 61701343, and 61771294).

\section{References}

[1] K. Szlachányi and P. Vecsernyés, "Quantum symmetry and braid group statistics inG-spin models," Communications in Mathematical Physics, vol. 156, no. 1, pp. 127-168, 1993.

[2] L. N. Jiang and M. Z. Guo, "The Galois correspondence in field algebra of G-spin model," Acta Mathematica Sinica, English Series, vol. 21, no. 4, pp. 673-680, 2005.

[3] Q. L. Xin and L. N. Jiang, "Symmetric structure of field algebra of G-spin models determined by a normal subgroup," Journal of Mathematical Physics, vol. 55, Article ID 091703, 2014.

[4] F. Nill and K. Szlachányi, "Quantum chains of Hopf algebras with quantum double cosymmetry," Communications in Mathematical Physics, vol. 187, no. 1, pp. 159-200, 1997.

[5] T. Q. Cao, Q. L. Xin, X. M. Wei, and L. N. Jiang, "Jones type basic construction on Hopf spin models," Mathematics, vol. 8, no. 9, p. 1547, 2020.

[6] S. Montgomery, Hopf Algebras And Their Actions On Rings, American Mathematical Society, Providence, RI, USA, 1993.

[7] M. E. Sweedler, Hopf Algebras, W.A. Benjamin, New York, NY, USA, 1969.

[8] V. G. Drinfel'd, Quantum Groups, pp. 798-820, Academic Press, Cambridge, UK, 1986.

[9] T. H. Koornwinder and N. M. Muller, "The quantum double of a (locally) compact group," Lie Theory, vol. 7, pp. 101-120, 1997.

[10] S. Majid, "Quantum double for quasi-Hopf algebras," Letters in Mathematical Physics, vol. 45, pp. 1-9, 1998.

[11] F. Hausser and F. Nill, "Diagonal crossed products by duals of quasi-quantum groups," Reviews in Mathematical Physics, vol. 11, no. 05, pp. 553-629, 1999.

[12] F. Hausser and F. Nill, "Doubles of quasi-quantum groups," Communications in Mathematical Physics, vol. 199, no. 3, pp. 547-589, 1999.

[13] D. Bulacu and S. Caenepeel, "The quantum double for quasitriangular quasi-Hopf algebras," Communications in Algebra, vol. 31, no. 3, pp. 1403-1425, 2003.

[14] L. Delvaux and A. Van Daele, "The Drinfel'd double of multiplier Hopf algebras," Journal of Algebra, vol. 272, no. 1, pp. 273-291, 2004. 
[15] L. L. Chen, Quivers and Hopf Algebra, Zhejiang University, Zhejiang, China, Ph.D. Disseration, 2008.

[16] A. V. Daele, "The Haar measure on finite quantum groups," Proceedings of the American Mathematical Society, vol. 125, no. 12, pp. 3489-3500, 1997.

[17] B. R. Li, Operator Algebras, Scientific Press, Beijing, China, 1998.

[18] N. Andruskiewitsch and J. Devoto, "Extensions of Hopf algebras," St. Petersburg Mathematical Journal, vol. 7, no. 1, pp. 17-52, 1996.

[19] S. Burciu, "Normal coideal subalgebras of semisimple Hopf algebras," Journal of Physics: Conference Series, vol. 346, Article ID 012004, 2012.

[20] A. Chirvasitu, P. Kasprzak, and P. Szulim, "Integrals in left coideal subalgebras and group-like projections," Algebras and Representation Theory, vol. 23, no. 4, pp. 1499-1522, 2020.

[21] S. Burciu, "Kernels of representations and coideal subalgebras of Hopf algebras," Glasgow Mathematical Journal, vol. 54, no. 1, pp. 107-119, 2012.

[22] R. G. Larson and D. E. Radford, "Finite dimensional cosemisimple Hopf algebras in characteristic 0 are semisimple," Journal of Algebra, vol. 117, no. 2, pp. 267-289, 1988.

[23] J. Dimier, C $C^{*}$-Algebra, North-Holland, Amsterdam, 1977. 